\title{
Schizophrenia-associated dysbindin modulates axonal mitochondrial movement in cooperation with $\mathrm{p} 150^{\text {glued }}$
}

\author{
Bo Kyoung Suh', Seol-Ae Lee1, Cana Park ${ }^{1,2}$, Yeongjun Suh', Soo Jeong Kim, Youngsik Woo ${ }^{1}$, \\ Truong Thi My Nhung ${ }^{1}$, Su Been Lee ${ }^{1}$, Dong Jin Mun ${ }^{1}$, Bon Seong Goo ${ }^{1}$, Hyun Sun Choi ${ }^{1,3}$, So Jung Kim \\ and Sang Ki Park ${ }^{1 *}$
}

\begin{abstract}
Mitochondrial movement in neurons is finely regulated to meet the local demand for energy and calcium buffering. Elaborate transport machinery including motor complexes is required to deliver and localize mitochondria to appropriate positions. Defects in mitochondrial transport are associated with various neurological disorders without a detailed mechanistic information. In this study, we present evidence that dystrobrevin-binding protein 1 (dysbindin), a schizophrenia-associated factor, plays a critical role in axonal mitochondrial movement. We observed that mitochondrial movement was impaired in dysbindin knockout mouse neurons. Reduced mitochondrial motility caused by dysbindin deficiency decreased the density of mitochondria in the distal part of axons. Moreover, the transport and distribution of mitochondria were regulated by the association between dysbindin and p150 glued. Furthermore, altered mitochondrial distribution in axons led to disrupted calcium dynamics, showing abnormal calcium influx in presynaptic terminals. These data collectively suggest that dysbindin forms a functional complex with p150glued that regulates axonal mitochondrial transport, thereby affecting presynaptic calcium homeostasis.
\end{abstract}

Keywords: Mitochondrial movement, Calcium homeostasis, Dysbindin, p150 glued, Dynactin complex

\section{Introduction}

Neurons harbor several hundred mitochondria along their projections because of their massive energy demand and calcium homeostasis. Proper distribution of mitochondria is achieved by bidirectional mitochondrial movement along microtubule-based tracks through collaborations of various motor proteins (such as dynein and kinesin) and motor adaptors $[1,2]$.

Dynactin is a multi-subunit protein complex required for dynein-mediated transport of cargos, including vesicles and organelles, along the microtubule. $\mathrm{p} 150^{\text {glued }}$, also known as dynactin 1 , is crucial for dynein motor activity

\footnotetext{
*Correspondence: skpark@postech.ac.kr

${ }^{1}$ Department of Life Sciences, Pohang University of Science and Technology, Pohang, Republic of Korea

Full list of author information is available at the end of the article
}

while the other subunits maintain the structure of the complex [3, 4]. p150 glued recruits dynein to the plus ends of microtubules and membranous cargos, allowing the motor proteins to achieve long-distance and processive transport along the microtubules [5, 6]. Animal models with disrupted dynein-dynactin complex exhibit symptoms of neurodegenerative diseases, including motor neuron degeneration. Cytoskeleton-associated protein glycine-rich (CAP-Gly) domain of $150^{\text {glued }}$ is essential for the microtubule-binding affinity of $\mathrm{p} 150^{\text {glued }}$ and mutations in this domain cause defects in spinal neurons [7]. Similarly, mutations in $\mathrm{p} 150^{\text {glued }}$ have been reported in patients with neurodegenerative diseases, such as spinobulbar muscular atrophy (SBMA) and amyotrophic lateral sclerosis (ALS) [8]. original author(s) and the source, provide a link to the Creative Commons licence, and indicate if changes were made. The images or other third party material in this article are included in the article's Creative Commons licence, unless indicated otherwise in a credit line to the material. If material is not included in the article's Creative Commons licence and your intended use is not permitted by statutory regulation or exceeds the permitted use, you will need to obtain permission directly from the copyright holder. To view a copy of this licence, visit http://creativecommons.org/licenses/by/4.0/. The Creative Commons Public Domain Dedication waiver (http://creativeco mmons.org/publicdomain/zero/1.0/) applies to the data made available in this article, unless otherwise stated in a credit line to the data. 
Defects in the mitochondrial transport are also related to a considerable number of neurological disorders. Notably, the impaired axonal mitochondrial transport in Alzheimer's disease and Parkinson's disease are related to accumulations of amyloid $\beta(A \beta)$ and the higher level of $\alpha$-synuclein aggregation, respectively $[9,10]$. Reduced mitochondrial density in soma and axon terminals were observed in patients with schizophrenia [11-13]. In addition, abnormal intracellular calcium homeostasis, regulated by mitochondria and endoplasmic reticulum (ER), is implicated in schizophrenia [14-17]. However, the precise mechanistic basis of the link has not been clearly elucidated.

Dystrobrevin-binding protein 1 (dysbindin) is a coiled-coil-containing protein that plays diverse roles in neuronal morphogenesis, synaptic vesicle biogenesis, exocytosis, and dendritic spinogenesis [18-22]. The association of dysbindin and schizophrenia was revealed by human postmortem studies of schizophrenic patients showing decreased expression levels of dysbindin in the brain and impaired cognitive function [23-25]. Dysbindin-knockout mice (Sandy) show abnormalities in dendritic morphogenesis, long-term potentiation, and synaptic transmission [26, 27], along with schizophrenia-like behavioral phenotypes [28-30]. Besides, some studies including a proteomics analysis of dysbindin suggested its potential association with motor complexes [31-33]. Given that dysbindin was detected in the vicinity of microtubules and outer membranes of mitochondria concurrently [31], we hypothesized dysbindin might play a role in mitochondrial movement along microtubules, which can provide mechanistic insight into schizophrenia-related synaptic functions.

In this study, we newly demonstrate that dysbindin functions as a regulator of neuronal mitochondrial motility and distribution, which is achieved by functional coordination of dynactin motor complex. We also show the significance of this process for the local calcium homeostasis in the neuron.

\section{Results \\ Dysbindin regulates the mitochondrial movement and distribution in axons}

To identify the regulatory function of dysbindin in the movement of mitochondria, we measured axonal transport of mitochondria in primary cultured cortical neurons from wild-type (WT) and Sandy mouse embryos. Mitochondrial motility represented by the fraction of motile axonal mitochondria in Sandy neurons decreased significantly relative to that in WT neurons (Fig. 1a, b). The defects were effectively reversed by the overexpression of dysbindin, indicating that the defects in mitochondrial movement are specific to dysbindin deficiency. When the directionality of axonal movement of mitochondria was analyzed, the anterograde movement decreased significantly whereas the retrograde movement remained intact (Fig. 1c).

To determine whether the defects in mitochondrial motility are extended to the mitochondrial distribution in neurons, we examined mitochondrial density along the axon. We selected two axonal areas for timelapse imaging: a $150 \mu \mathrm{m}$ region proximal to the soma (proximal axon) and a $150 \mu \mathrm{m}$ region from the axon tip, including a growth cone (distal axon). As a result, while the mitochondrial density in the proximal axons of the Sandy neurons was not altered, the distal axons in the Sandy neurons displayed a significant decrease in density that was effectively reversed by dysbindin overexpression (Fig. 1d). These results were recapitulated in the short hairpin ribonucleic acid (shRNA)-based dysbindin knockdown experiment. The knockdown effects were reversed by shRNA-resistant dysbindin co-expression, suggesting that the alterations in mitochondrial motility in the proximal axons and mitochondrial density in the distal axons were also specific to dysbindin depletion (Additional file 1: Fig. S1a-c). In agreement with the above results, dysbindin overexpression showed an increase in the fraction of motile mitochondria (Additional file 1: Fig. S1d-f). The effect of dysbindin on mitochondrial motility and density was mostly intact in dendrites (Additional file 1: Fig. S1g, h), collectively suggesting that dysbindin is an important regulator in axonal mitochondrial transport.

\section{Dysbindin interacts with $\mathrm{p} 150^{\text {glued }}$ to regulate its microtubule association}

To identify the mechanistic link between dysbindin and motor complexes relevant to the mitochondrial transport, we first tested the physical association between dysbindin and $\mathrm{p} 150^{\text {glued }}$, a strong candidate motor protein to interact with dysbindin [33]. Dysbindin was co-immunoprecipitated with $\mathrm{p} 150^{\text {glued }}$ in human embryonic kidney (HEK) 293 cell lysates (Fig. 2a). We next investigated the subcellular localization of dysbindin to mitochondria by immunocytochemistry. We found that dysbindin signals overlapped with those of MTS-mCherry, a mitochondrial marker, in axons of primary cortical neurons (Fig. 2b). Moreover, a significant portion of endogenous dysbindin was detected in the mitochondrial fraction obtained from primary cultured neurons (Fig. 2c). Consistently, a substantial level of endogenous dysbindin protein was detected in the mitochondrial fraction of mouse brain lysates (Additional file 2: Fig. S2a). The level of p150 glued associated with mitochondria in the WT and Sandy mice brains were not different significantly (Additional file 2: Fig. S2b). 

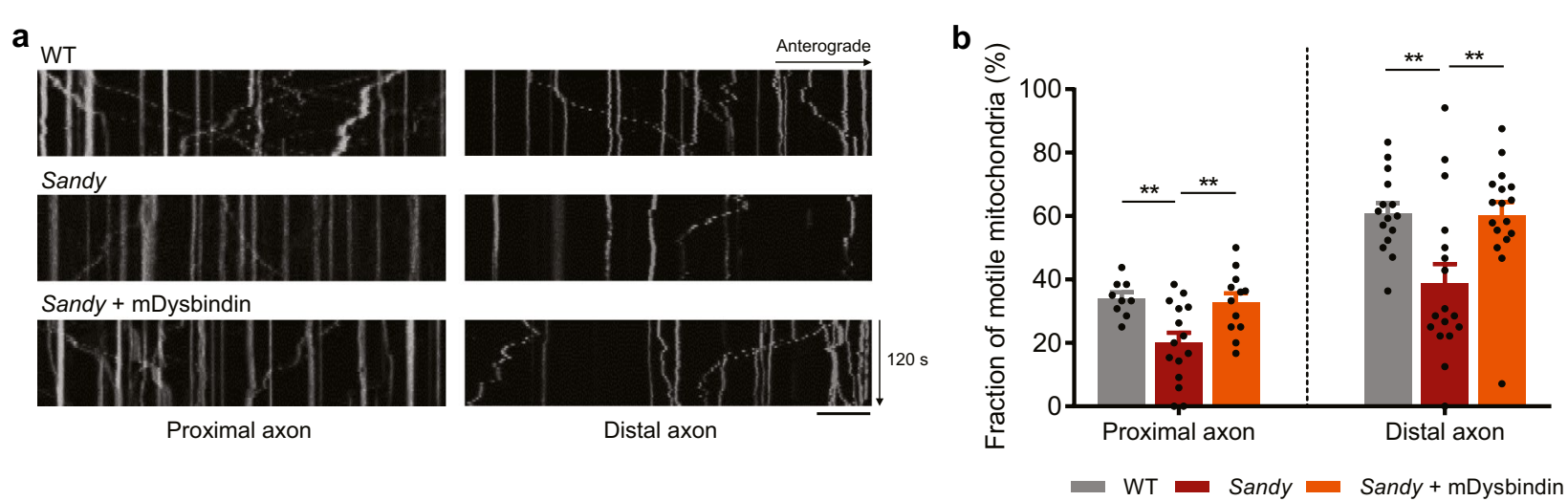

C

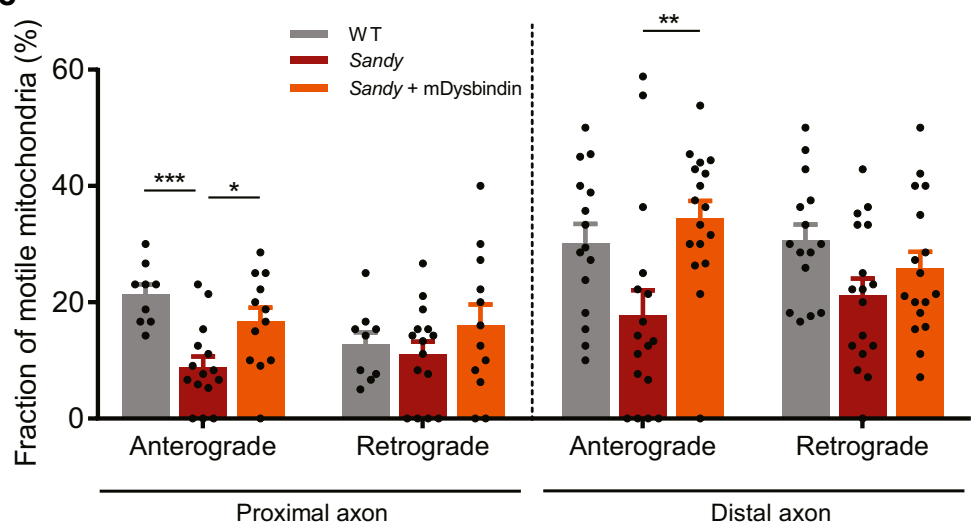

d

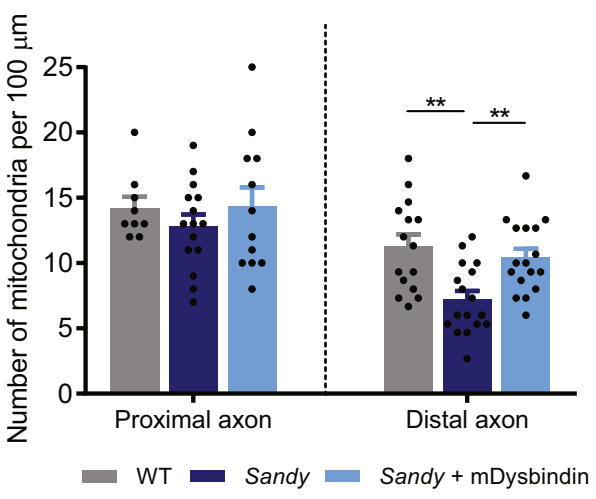

Fig. 1 Dysbindin regulates axonal mitochondrial movement and density. a Representative kymographs of mitochondrial movement in DIV 10-12 primary cortical neurons of WT and Sandy mice. The movements of mitochondria in the proximal and distal axons are shown. A scale bar represents $20 \mu \mathrm{m}$. b Quantitative analyses of the motile fraction of mitochondria, and $\mathbf{c}$ anterograde and retrograde movements of mitochondria. d Quantification of mitochondrial density (the number of mitochondria per $100 \mu \mathrm{m}$ ) in the proximal and distal axons of DIV 10-12 primary cortical neurons ( $n=9$ axons for WT, $n=15$ axons for Sandy, and $n=12$ axons for Sandy + mDysbindin for the proximal axons, $n=15$ axons for WT, $n=17$ axons for Sandy, and $n=17$ axons for Sandy + mDysbindin for the distal axons). All results are presented as the mean $\pm S E M$. ${ }^{*} p<0.05,{ }^{* *} p<0.01$, and ${ }^{* * *} p<0.001$ from one-way ANOVA with Bonferroni's multiple comparison test

In the immunocytochemical analysis with primary cortical neurons, the dysbindin signal was co-localized with p150 ${ }^{\text {glued }}$ along the axonal microtubule (Fig. 2d), supporting the notion that dysbindin is a microtubular protein participating in the cargo transport. Based on the potential involvement of dysbindin in microtubule-related processes $[34,35]$, we tested whether the association of p150 ${ }^{\text {glued }}$ with microtubules is affected by dysbindin. We performed a microtubule co-sedimentation assay using mouse brain lysates and observed a decrease in co-sedimentation of p150 glued and microtubules in Sandy mouse brain tissue compared to that of WT (Fig. 2e, f). The co-sedimentation of dynein intermediate chain (dynein IC), which directly binds to $150^{\text {glued }}$, was not affected (Fig. 2g).

Next, to test whether dysbindin affects the integrity of dynein-dynactin motor complex, we examined the interaction between $\mathrm{p} 150^{\text {glued }}$ and dynein IC. We observed a significant increase in the co-precipitation of p150 ${ }^{\text {glued }}$ and dynein IC upon dysbindin overexpression (Fig. 2h, i). Besides, p150 ${ }^{\text {glued }}$ co-immunoprecipitated with dynamitin (dynactin 2) significantly increased upon dysbindin overexpression (Fig. 2j, k). When dysbindin was knocked down, endogenous dynamitin co-immunoprecipitated with $\mathrm{p} 150^{\text {glued }}$ was also reduced (Additional file 2: Fig. S2c-f). These results indicate that dysbindin participates in the regulation of motor complex integrity as well as the interaction between individual dynactin subunits.

\section{Dysbindin regulates axonal mitochondrial motility in cooperation with $\mathrm{p} 150^{\text {glued }}$}

To identify the functional relationship between dysbindin and $\mathrm{p} 150^{\text {glued }}$ in mitochondrial transport, we 


\begin{abstract}
(See figure on next page.)
Fig. 2 Dysbindin interacts with $150^{\text {glued }}$ affecting microtubule association and complex integrity. a Co-immunoprecipitation of dysbindin and p150glued. Lysates from HEK293 cells transfected with GFP-hDysbindin and FLAG-p150 glued constructs were immunoprecipitated with anti-FLAG antibody. Immunoprecipitates were analyzed by western blotting with anti-GFP and anti-FLAG antibodies. b Co-localization of dysbindin and mitochondria in the axon of DIV 10 primary cortical neurons. Neurons transfected with GFP-mDysbindin and MTS-mCherry were stained with anti-GFP antibody. Overlapped signals are indicated by arrow heads. Scale bars represent $20 \mu \mathrm{m}$ (left) and $5 \mu \mathrm{m}$ (right). c Endogenous dysbindin detected from the mitochondrial fraction of DIV 11 primary cultured neurons. Dysbindin was detected by western blotting with anti-dysbindin antibody. Mitofilin and a-tubulin were used as markers for mitochondrial and cytosolic fractions, respectively. $\mathbf{d}$ Co-localization of dysbindin and $150^{\text {glued }}$ along the microtubules in the axon of DIV 10 primary cortical neurons. Neurons transfected with mRFP-C1, FLAG-mDysbindin and GFP-p150glued were stained with anti-FLAG (red) and anti-a-tubulin (blue) antibodies. Scale bars represent $20 \mu \mathrm{m}$ (left) and $5 \mu \mathrm{m}$ (right). e Microtubule co-sedimentation assay for p150 glued and dynein IC in mouse brain tissue. Polymerized tubulin in the pellet fraction (P) and non-polymerized tubulin in the supernatant (S) were subjected to western blotting with anti-p150glued and anti-dynein IC antibodies. $\mathbf{f}$ Quantification of the protein level of $150^{\text {glued }}$ and $\mathbf{g}$ the protein level of dynein IC in the pellet fraction normalized by that of a-tubulin. $\mathbf{h}$ Co-immunoprecipitation of $\mathrm{p} 150^{\text {glued }}$ and dynein IC upon dysbindin co-expression. Lysates from HEK293 cells transfected with indicated constructs were immunoprecipitated with anti-GFP antibody. Immunoprecipitates were analyzed by western blotting with anti-FLAG and anti-GFP antibodies. i Quantification of the protein level of co-immunoprecipitated p $150^{\text {glued }}$ normalized by immunoprecipitated dynein IC. j Co-immunoprecipitation of p150glued and dynamitin upon dysbindin co-expression. Lysates from HEK293 cells transfected with indicated constructs were immunoprecipitated with anti-FLAG antibody. Immunoprecipitates were analyzed by western blotting with anti-GFP and anti-FLAG antibodies. $\mathbf{k}$ Quantification of the protein level of co-immunoprecipitated p150 glued normalized by immunoprecipitated dynamitin. Asterisks indicate the protein of interest. All results are presented as the mean \pm SEM. ${ }^{*} p<0.05,{ }^{* *} p<0.01$, and ${ }^{* * *} p<0.001$ from Student's $t$ test
\end{abstract}

analyzed whether dysbindin-mediated mitochondrial movement and distribution are altered by $150^{\text {glued }}$ depletion. In $150^{\text {glued }}$ knockdown, mitochondrial motility in the proximal axons was significantly reduced (Fig. 3a, b) and the density of mitochondria in the distal axons was also significantly reduced (Fig. 3c), as seen in the dysbindin depletion conditions. Moreover, additional impairment of mitochondrial motility in the proximal axons (Fig. 3b) or mitochondrial density in the distal axons (Fig. 3c) was not observed following the concomitant knockdown of $\mathrm{p} 150^{\text {glued }}$ and dysbindin. Likewise, dysbindin overexpression did not restore the decrease in mitochondrial movement in the proximal axons or mitochondrial density in the distal axons caused by p150 $0^{\text {glued }}$ knockdown (Fig. 3d-f). The results from mitochondrial density in the proximal axons and mitochondrial motility in the distal axons are shown in 'Additional file 3: Fig. S3'. These data collectively indicate that dysbindin and $\mathrm{p} 150^{\text {glued }}$ cooperate to regulate mitochondrial transport in the same motor machinery.

To determine a functional dysbindin domain involved in association with $\mathrm{p} 150^{\text {glued }}$, we generated deletion mutants of dysbindin on the basis of domain predictions. Mostly, dysbindin interacts with binding partners via its coiled-coil structure, thus, we divided dysbindin protein into three parts, with the coiled-coil domain in the middle. The $\mathrm{N}$-terminal region (1-87 aa) lies from the first amino acid to the beginning of the coiledcoil domain, the middle region (88-180 aa) covers the coiled-coil domain and a leucine zipper motif, and the C-terminal region (181-351 aa) contains a dysbindin domain and a PEST domain (Fig. 3g) [36-38]. In coimmunoprecipitation analysis using these dysbindin fragments, only the $\mathrm{N}$-terminal region of dysbindin was co-immunoprecipitated with p150 glued (Fig. 3h). Consistently, the $\mathrm{N}$-terminal region of dysbindin restored the mitochondrial movement impaired upon dysbindin knockdown, whereas it was not the case for dysbindin lacking the $\mathrm{N}$-terminal region (Fig. $3 \mathrm{i}, \mathrm{j}$ ). These results suggest that the physical interaction between dysbindin and $\mathrm{p} 150^{\text {glued }}$ is important for regulating mitochondrial transport.

\section{Dysbindin controls calcium homeostasis in presynaptic terminals}

In the presynaptic terminal of axons, mitochondria regulate local calcium homeostasis [39, 40]. Therefore, we tested if the attenuated motility of mitochondria and the altered distribution of mitochondria in the distal axons result in altered local calcium dynamics. We performed time-lapse live-cell imaging using cyto-GCaMP6 in the axon tip of primary cultured cortical neurons after dysbindin knockdown (Fig. 4a, b). Potassium chloride $(\mathrm{KCl})$-stimulated axon terminal calcium influx increased in dysbindin-deficient neurons (Fig. 4c), whereas the amount of accumulated calcium did not change (Fig. 4d). Moreover, the decay time to half-maximum decreased in dysbindin-deficient neurons, showing faster off rates compared to the control neurons (Fig. 4e). These results were recapitulated in the comparison of calcium dynamics between WT and Sandy neurons, although the difference of the peak amplitudes between the two groups did not achieve statistical significance (Additional file 4: Fig. S4a-d). Increased calcium influx shown in the axon tip of dysbindin-deficient neurons was also reproduced in presynaptic boutons of dysbindin-deficient neurons 

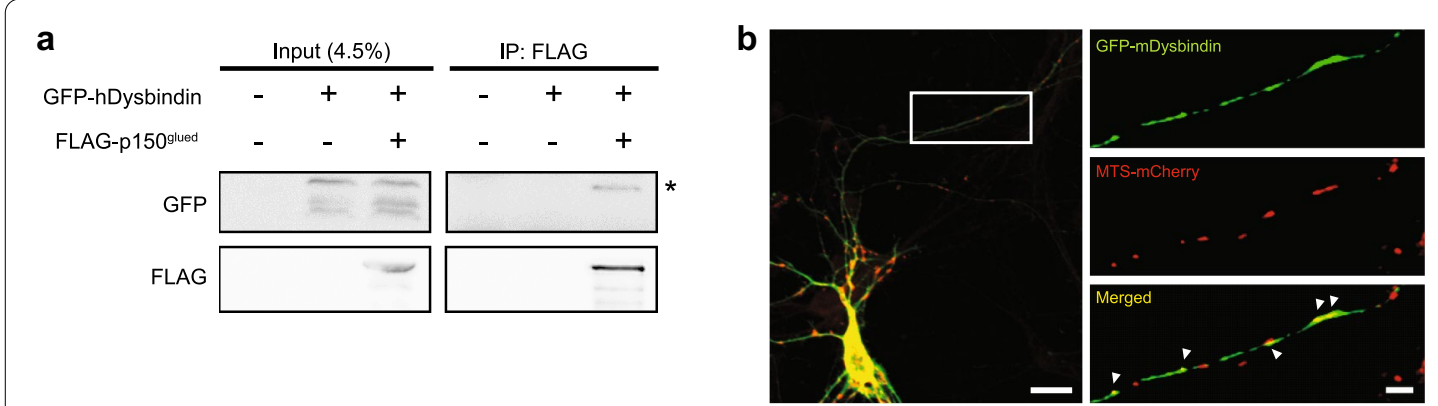

C
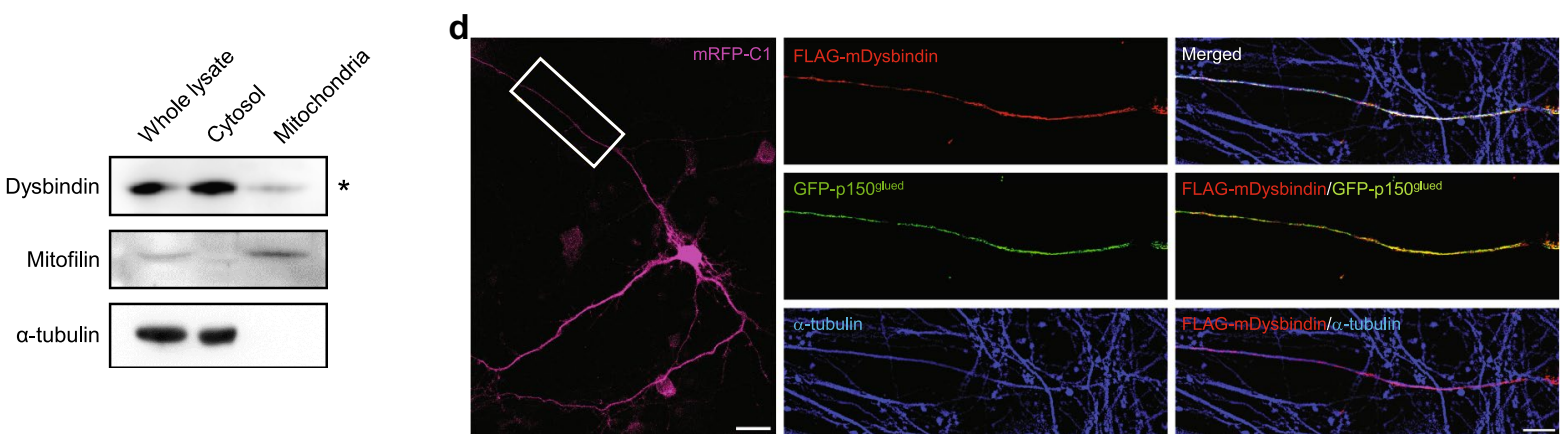

e
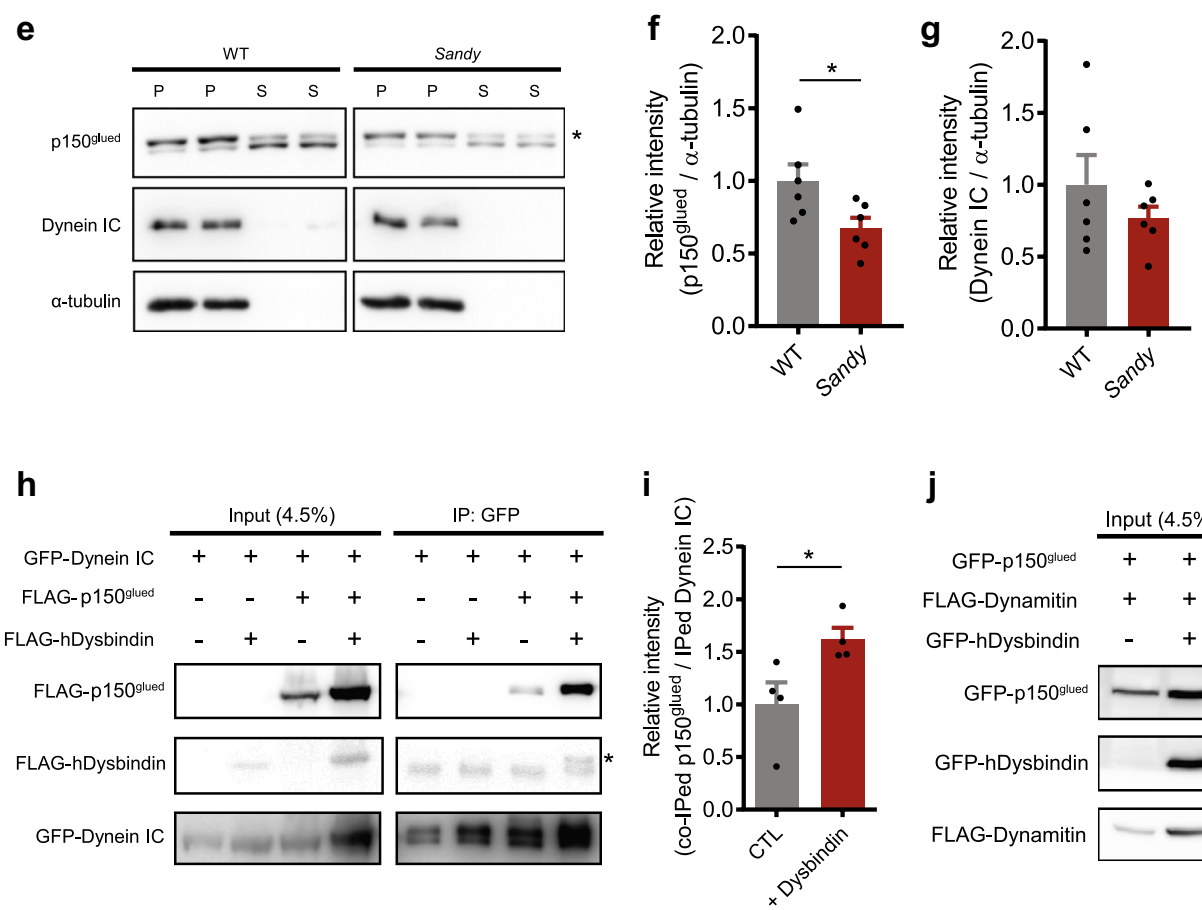

j
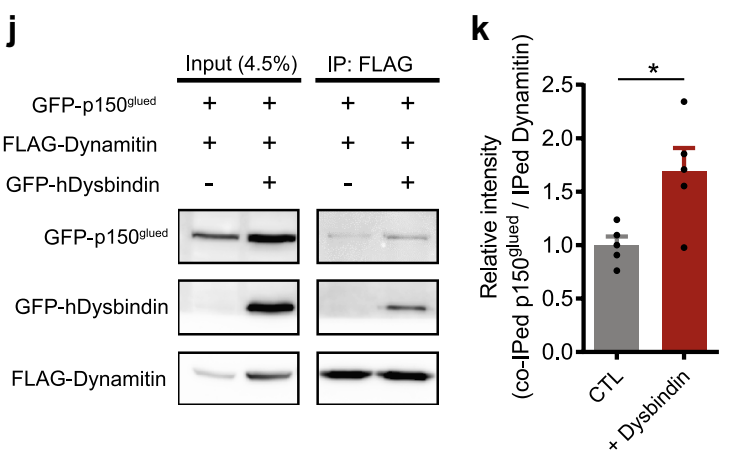

expressing vGlut1-GCaMP5G, a presynaptic calcium sensor (Additional file 4: Fig. S4e-g). Collectively, it is likely that impaired presynaptic calcium buffering is caused by the reduced number of mitochondria resulting from dysbindin deficiency, further highlighting the critical role of dysbindin in synaptic activity. 


\begin{abstract}
(See figure on next page.)
Fig. 3 Dysbindin regulates axonal mitochondrial motility in cooperation with p150 glued. a Representative kymographs of mitochondrial movement in the proximal axons of DIV 10-12 primary cortical neurons transfected as indicated. $\mathbf{b}$ Quantitative analysis of the motile fraction of mitochondria in the proximal axons ( $n=32$ axons for control (CTL) shRNA, $n=33$ axons for p150glued shRNA, $n=14$ axons for Dysbindin shRNA, and $n=24$ axons for p150 glued shRNA + Dysbindin shRNA). c Quantification of mitochondrial density (the number of mitochondria per $100 \mu \mathrm{m}$ ) in the distal axons of DIV 10-12 primary cortical neurons ( $n=26$ axons for CTL shRNA, $n=12$ axons for p150glued shRNA, $n=9$ axons for Dysbindin shRNA, and $n=17$ axons for p150 glued shRNA + Dysbindin shRNA). $\mathbf{d}$ Representative kymographs of mitochondrial movement in the proximal axons of DIV 10-12 primary cortical neurons transfected as indicated. e Quantitative analysis of the motile fraction of mitochondria in the proximal axons ( $n=34$ axons for CTL shRNA, $n=30$ axons for p150 glued shRNA, $n=32$ axons for mDysbindin, and $n=33$ axons for $p 150^{\text {glued }}$ shRNA + mDysbindin). $\mathbf{f}$ Quantification of mitochondrial density (the number of mitochondria per $100 \mu \mathrm{m}$ ) in the distal axons of DIV 10-12 primary cortical neurons ( $n=49$ axons for CTL shRNA, $n=27$ axons for p150 glued shRNA, $n=34$ axons for mDysbindin, and $n=30$ axons for p150glued shRNA + mDysbindin). $\mathbf{g}$ Schematic diagrams of deletion mutants of dysbindin protein used for the co-immunoprecipitation analysis. aa, amino acids. $\mathbf{h}$ Co-immunoprecipitation of $150^{9 \text { lued }}$ with dysbindin fragments. Lysates from HEK293 cells transfected with FLAG-p150glued and indicated GFP-hDysbindin fragments were immunoprecipitated with anti-FLAG antibody. Immunoprecipitates were analyzed by western blotting with anti-GFP and anti-FLAG antibodies. i Representative kymographs of mitochondrial movement in the proximal axons of DIV 10-12 primary cortical neurons transfected as indicated. $\mathbf{j}$ Quantitative analysis of the motile fraction of mitochondria in the proximal axons ( $\mathrm{n}=15$ axons for CTL shRNA, $n=18$ axons for Dysbindin shRNA + Control vector (pFLAG-CMV2), $n=20$ axons for Dysbindin shRNA + mDysbindin WT, $n=24$ axons for Dysbindin shRNA + mDysbindin 1-87 aa, and $n=9$ axons for Dysbindin shRNA + mDysbindin 88-352 aa). Scale bars represent $20 \mu$ m. All results are presented as the mean \pm SEM. ${ }^{*} p<0.05,{ }^{* *} p<0.01$, and ${ }^{* * *} p<0.001$ from one-way ANOVA with Bonferroni's multiple comparison test
\end{abstract}

\section{Discussion}

In this study, we demonstrate that dysbindin regulates axonal mitochondrial movement in cooperation with dynactin complex. Dysbindin deficiency causes defects in mitochondrial transport along the axon and results in reduced mitochondrial density in the distal axons. Finally, it affects the local calcium homeostasis in axon terminals, resulting in abnormal calcium influx (Fig. 4f).

We proposed a novel functional link between dysbindin and the microtubule motor complex by multiple experimental approaches. Indeed, previous EM-based study has shown the existence of dysbindin in the vicinity of microtubule structure [31], hinting at their physical and functional association. Extending this observation, we demonstrated that the association of $150^{\text {glued }}$ with microtubules was significantly decreased in the Sandy mice. As $\mathrm{p} 150^{\text {glued }}$ is required to recruit dynein motor to microtubules for the efficient initiation of transport and its processivity $[5,6]$, our results indicate that dysbindin plays a role as a motor adaptor, recruiting dyneindynactin motor complex to microtubules in axons. Given that $\mathrm{p} 150^{\text {glued }}$ in mitochondrial fraction was largely not changed in Sandy mice (Additional file 2: Fig. S2b), the function of dysbindin seems to be specific for the association of dynein-dynactin complex to microtubules but irrelevant to its association to cargos. In this regard, dysbindin may function as a novel adaptor or scaffolding protein, modulating the recruitment of dynein-dynactin complex to the microtubule and subsequently determining the efficiency of organelle and vesicle transports along the axon.

Dysbindin is a component of biogenesis of lysosomerelated organelle complex 1 (BLOC-1) and a large number of studies on dysbindin have focused on its function as a constituent of BLOC-1 complex [41]. In particular, BLOC-1 and dysbindin are involved in transporting or positioning multiple targets in neurons. Dysbindin functions in vesicle transport mediated by BLOC- 1 and adaptor related protein complex-3 (AP-3) for sorting cargos from cell bodies to nerve terminals [42]. Specific subunits of BLOC-1, such as snapin, regulate lysosome trafficking along the microtubule by recruiting motor machinery to lysosomes [43]. Therefore, it would be interesting to see whether dysbindin and $\mathrm{p} 150^{\text {glued }}$ cooperate to transport other cargos beyond mitochondria, such as lysosomes and synaptic vesicles, functioning as a general factor for many more cargos.

Dysbindin-deficient neurons showed an excessive calcium influx in the presynaptic axon tip (Fig. 4c). Recent reports support the notion that presynaptic calcium buffering is affected by the presence of mitochondria along with the attenuation of presynaptic calcium transients in response to action potentials and downregulation of synaptic vesicle release $[44,45]$. Indeed, we demonstrated that dysbindin deficiency leads to a decreased local mitochondrial density in the axon tip (Fig. 1d), which likely to underlie the exaggerated local calcium increase. Lines of evidence have shown that dysbindin-deficient mice show decreased glutamate release [46, 47] and GABAergic transmission $[48,49]$ in the prefrontal cortex, which all are interlinked with presynaptic calcium transients. In addition, this impaired neurotransmission has been implicated in the etiology of schizophrenia [29, 50, 51]. Further work is required to identify whether the dysbindin-mediated regulation of calcium homeostasis is on the same line with previously known presynaptic functions of dysbindin mediating the trafficking and priming of synaptic vesicles. 


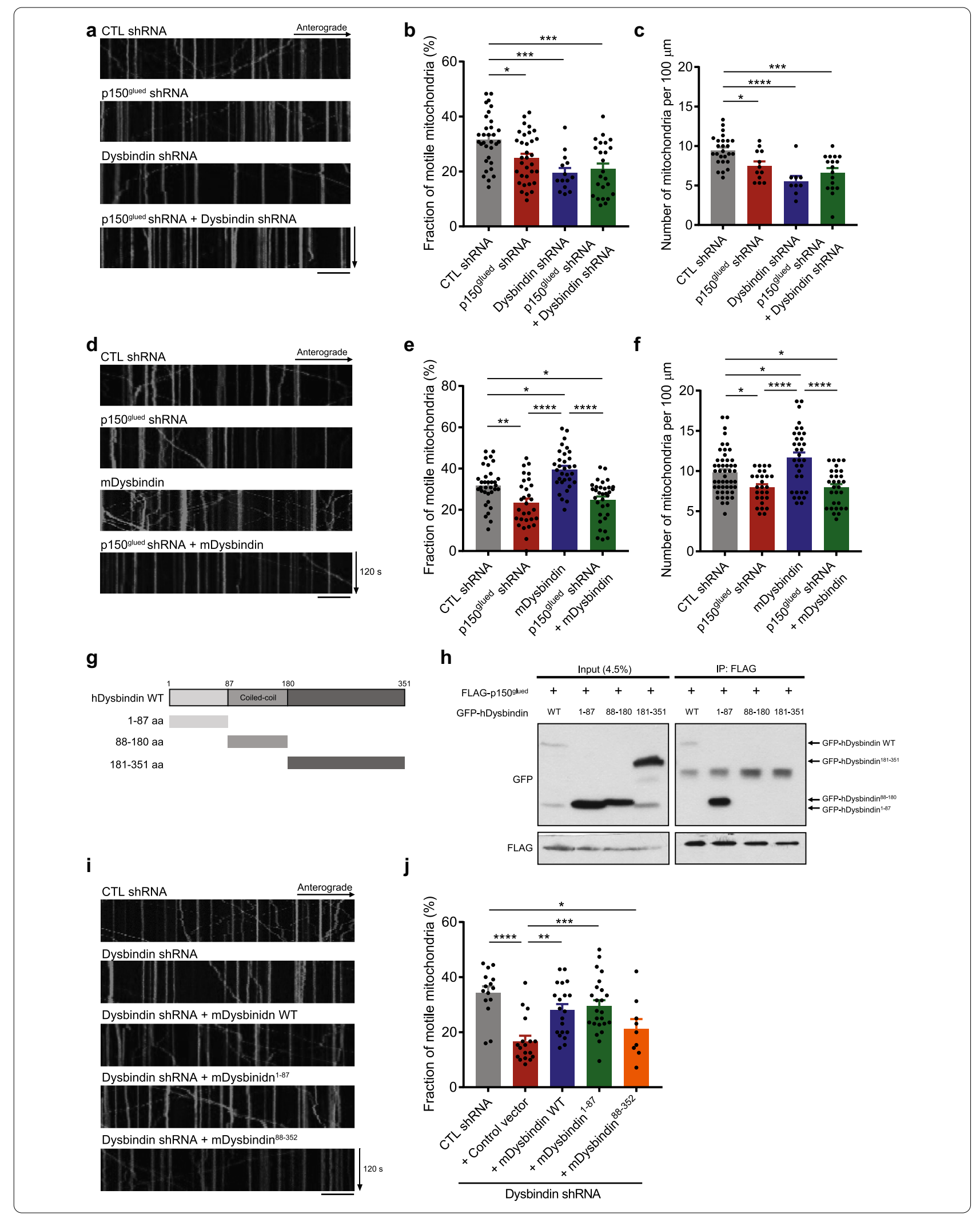




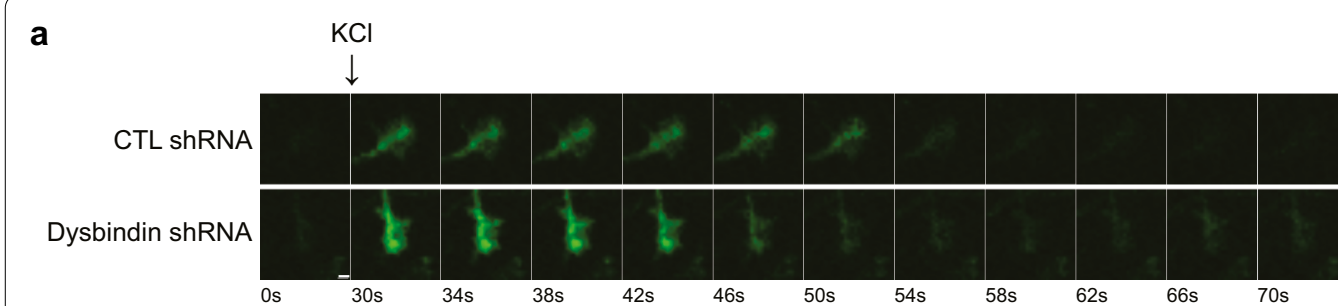

b

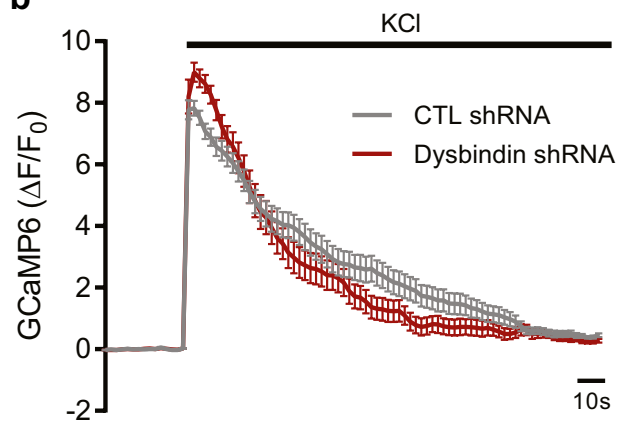

f

C

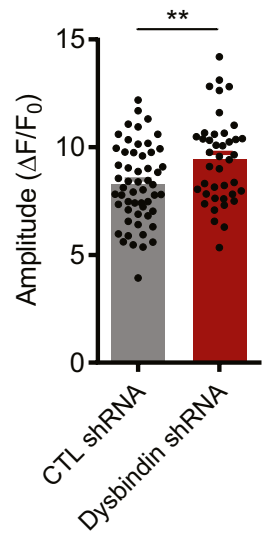

d

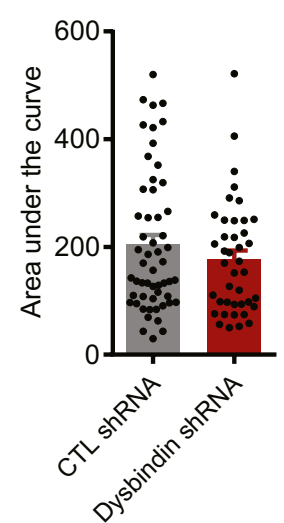

e

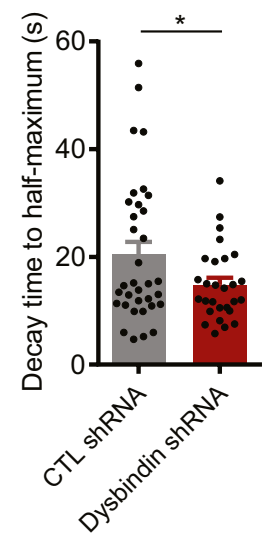

Dysbindin +
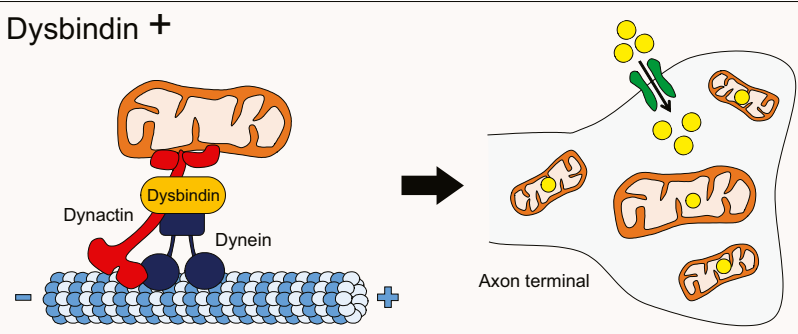

Dysbindin -

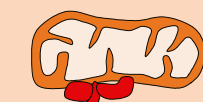

Dynactin

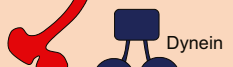

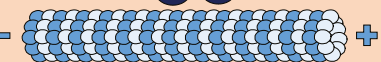

Mitochondrial motility $\downarrow$

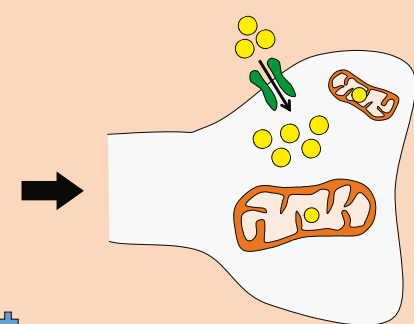

Mitochondrial density $\downarrow$ Abnormal calcium homeostasis

Gro Mitochondrion Microtubule $\bigcirc$ calcium ion

Fig. 4 Dysbindin mediates calcium homeostasis in presynaptic terminals. a Representative time-lapse images of calcium responses in the axon tip of DIV 10-12 primary cortical neurons transfected with the indicated shRNA. A scale bar represents $2 \mu \mathrm{m}$. b Calcium response graph obtained after $50 \mathrm{mM} \mathrm{KCl}$ stimulation. c Statistically analyzed peak amplitudes, $\mathbf{d}$ the area under the curves, and $\mathbf{e}$ the decay time to half-maximum of the calcium response graph ( $n=55$ for CTL shRNA and $n=41$ for Dysbindin shRNA). $\mathbf{f}$ A schematic model of the function of dysbindin in regulating mitochondrial movement. The association of dysbindin to dynactin complex facilitates the mitochondrial motility and thereby affects calcium dynamics in the axon tip. All results are presented as the mean \pm SEM. ${ }^{*} p<0.05,{ }^{* *} p<0.01$, and ${ }^{* * *} p<0.001$ from Student's t-test 
Altered mitochondrial distribution in neurons is implicated in the pathogenesis of multiple psychiatric conditions, including schizophrenia. Patients with schizophrenia show defects in the distribution of axonal mitochondria in their caudate nucleus and putamen, as well as a decreased mitochondrial density in the axon terminal region in their anterior cingulate cortex $[12,13]$. In this regard, the potential role of dysbindin-p150 ${ }^{\text {glued }}$ complex in the movement and distribution of mitochondria may explain one of the pathological mechanisms of schizophrenia. Previously, it was reported that dysbindin forms a functional complex with disrupted-in-schizophrenia 1 (DISC1), another schizophrenia susceptibility factor. Their physical interaction is important for stabilizing dysbindin and regulating neurite outgrowth [38]. In addition, DISC1 has been reported to regulate axonal mitochondrial movement by anchoring mitochondria with syntaphilin $[52,53]$. Therefore, it would be of immediate interest to investigate potential communications between dysbindin-containing motor complex and DISC1-containing mitochondrial anchoring machinery in the proper axonal mitochondrial positioning and related neurological disease conditions.

\section{Methods}

\section{Animals}

Sandy $(s d y-/-)$ mice on DBA/2J background were obtained from the Jackson Laboratory (Bar Harbor, ME) [54]. Mice in this study were on a C57BL/6 background, obtained by breeding the Sandy mice on DBA/2J background with $\mathrm{C} 57 \mathrm{BL} / 6$ mice for at least eight generations. Both C57BL/6 and Sandy mice were used for primary cortical neuron culture and brain lysate preparation. Pregnant C57BL/6 mice were purchased from Hyochang Science (Daegu, Republic of Korea). All animal procedures were approved by the Institutional Animal Care and Use Committee (IACUC) of Pohang University of Science and Technology (POSTECH-2019-0024). All experiments were carried out in accordance with the approved guidelines.

\section{Cell culture and transfection}

HEK293 cells were cultured in DMEM (HyClone) supplemented with $10 \%(\mathrm{v} / \mathrm{v})$ fetal bovine serum (FBS) (Gibco) and 1\% penicillin/streptomycin (Gibco). All cell lines were authenticated using STR profiling method and were tested negative for mycoplasma contamination. All cells were transfected by using transfection reagent either VivaMagic (Vivagen) or Lipofectamine 2000 (Thermo Fisher Scientific) according to the manufacturer's instructions.

Primary cultures of cortical neurons were established by isolating E15 mouse embryo cortical tissues in HBSS
(Gibco) and dissociating tissues in $0.25 \%$ trypsin (SigmaAldrich) and $0.1 \%$ DNase I (Sigma-Aldrich) for $10 \mathrm{~min}$ at $37{ }^{\circ} \mathrm{C}$. Cells were resuspended in neurobasal medium (Gibco) supplemented with $10 \mathrm{mM}$ HEPES pH 7.4 and $10 \%(\mathrm{v} / \mathrm{v})$ horse serum for final cell concentration being $3.0 \times 10^{5}$ cells $/ \mathrm{mL}$ and plated on glass coverslips precoated with poly-D-lysine and laminin. After $2 \mathrm{~h}$ of plating, cell medium was replaced to neurobasal medium containing $2 \mathrm{mM}$ glutamine, 2\% (v/v) B27 supplement (Gibco), and $1 \%(\mathrm{v} / \mathrm{v})$ penicillin/streptomycin. The neurons were transfected at days in vitro (DIV) 7-9 with Lipofectamine 2000 and the medium was replaced with the culture medium $4 \mathrm{~h}$ after transfection.

\section{Antibodies and plasmids}

Anti-dysbindin rabbit polyclonal antibody was a kind gift from Dr. Koh-ichi Nagata (Institute for Developmental Research, Japan) and was used as previously described [38, 55]. Anti-p150 ${ }^{\text {glued }}$ mouse monoclonal antibody (Cat\# 612709, BD bioscience), anti-dynein IC1/2, cytosolic mouse monoclonal (Cat\# sc-13524, Santa Cruz Biotechnology), anti-mitofilin rabbit polyclonal (Cat\# NB100-1919, Novus Biologicals), anti-FLAG rabbit polyclonal and mouse monoclonal (Cat\# F7425, and Cat\# F1804, respectively, Sigma-Aldrich), anti- $\alpha-$ tubulin mouse monoclonal (Cat\# 66031-1-Ig, Proteintech Group), anti-GAPDH mouse monoclonal (Cat\# sc-32233, Santa Cruz Biotechnology), anti-GFP rabbit polyclonal (Cat\# A-11122, Molecular Probes), and anti-GFP mouse monoclonal (Cat\# sc-9996, Santa Cruz Biotechnology) antibodies were used for immunoblotting, IP, and immunostaining experiments. For immunoblotting, HRPconjugated sheep anti-mouse IgG (Cat\# NA931, GE Healthcare) and donkey anti-rabbit IgG (Cat\# NA934, GE Healthcare) were used as secondary antibodies. VeriBlot for IP Detection Reagent (HRP) (Cat\# ab131366, Abcam) was also used for immunoblotting of IP. For immunostaining, Alexa Fluor 568 conjugated goat anti-rabbit IgG (Cat\# A-11011, Molecular Probes), Alexa Fluor 488 conjugated goat anti-mouse IgG (Cat\# A-11001, Molecular Probes), 647 conjugated goat anti-rabbit antibodies (Cat\# A-21245, Molecular Probes), and Alexa Fluor 405 conjugated goat anti-mouse IgG (Cat\# A-31553, Molecular Probes) were used as secondary antibodies.

Constructs for human dysbindin (isoform a, hDysbindin) were prepared by cloning into pFLAG-CMV2 (Sigma-Aldrich) and pEGFP-N1 (Clontech), and constructs for mouse dysbindin (mDysbindin) were prepared by cloning into pFLAG-CMV2, as previously described [38]. To construct the deletion mutants of dysbindin, the regions of human dysbindin and mouse dysbindin corresponding to the designated codons were amplified by PCR using GFP-hDysbindin or shRNA-resistant form of 
FLAG-mDysbindin as a template and inserted into the pEGFP-N1 or pFLAG-CMV2, respectively, as previously described [38]. Constructs for human p150 ${ }^{\text {glued }}$ (dynactin subunit 1 isoform 1 ) were cloned into pFLAG-CMV2 and pEGFP-N1, human dynamitin (dynactin subunit 2 isoform 1) was cloned into pFLAG-CMV2, and human dynein intermediate chain (dynein IC) was cloned into pEGFP-C3 (Clontech). Mitochondrial transit sequence (MTS)-mCherry was prepared as previously described [53], pGP-CMV-GCaMP6s was a gift from Douglas Kim and GENIE Project (Cat\# 40753) [56], and pCAG-vGlut1GCaMP5G was a kind gift from Dr. Seok-Kyu Kwon (Korea Institute of Science and Technology, Republic of Korea). The oligonucleotide sequences used for mouse dysbindin shRNA were GTGATAAGTCAAGAGAAG CTTCAAGAGAGCTTCTCTTGACTTACACTTTTTT, TCGAGAAAAAAGTGATAAGTCAAGAGAAGCTCT CTTGAAGCTTCTCTTGACTTATCACA, as previously described [38], and the sequence for human dysbindin shRNA was AAGTGACAAGTCAAGAGAAGC. The oligonucleotide sequences for mouse p150 ${ }^{\text {glued }}$ shRNA were GACTTCACCCCTTGATTAA and CGAGCTCACCAC AGACCTG [57]. These oligonucleotides were annealed and ligated into the pLentiLox3.7 vector using PstI and Xhol sites. The shRNA-resistant FLAG-mDysbindin construct was generated by site-directed mutagenesis, and the sequence for the PCR primer was AAGACTTTA AGTGACAAATCAAGGGAGGCAAAAGTGAAA as previously described [38].

\section{Immunoprecipitation and immunoblotting}

Transfected HEK293 cells were lysed in $1 \times$ ELB lysis buffer [50 mM Tris, pH 8.0, $250 \mathrm{mM} \mathrm{NaCl}, 0.1 \% \mathrm{NP}-40$, $5 \mathrm{mM}$ EDTA, $2 \mathrm{mM} \mathrm{NaPPi}, 10 \mathrm{mM} \mathrm{NaF}, 2 \mathrm{mM} \mathrm{Na}_{3} \mathrm{VO}_{4}$, $1 \mathrm{mM}$ DTT, and protease inhibitor cocktail (Roche)] and mouse brain tissues were homogenized and lysed in $1 \times$ NP40 lysis buffer (50 mM Tris, pH 8.0, $150 \mathrm{mM} \mathrm{NaCl}, 1 \%$ NP-40, 5 mM EDTA, $2 \mathrm{mM} \mathrm{NaPPi}, 10 \mathrm{mM} \mathrm{NaF}, 2 \mathrm{mM}$ $\mathrm{Na}_{3} \mathrm{VO}_{4}, 1 \mathrm{mM}$ DTT, and protease inhibitor cocktail).

For immunoprecipitation, the lysates were incubated with 1-2 $\mu \mathrm{g}$ of antibody at $4{ }^{\circ} \mathrm{C}$ overnight on a rocking platform. Protein-A agarose beads (Roche) resuspended in the lysis buffer were mixed with immunoprecipitated lysates and incubated for $2-3 \mathrm{~h}$ at $4{ }^{\circ} \mathrm{C}$ with constant rotation. The precipitates were washed three times with the lysis buffer and mixed with SDS sample buffer for immunoblotting.

For immunoblotting, proteins were denatured by mixing lysates with $5 \times$ SDS sample buffer (2\% SDS, $60 \mathrm{mM}$ Tris $\mathrm{pH} 6.8,24 \%$ glycerol, and $0.1 \%$ bromophenol blue, and $5 \% \beta$-mercaptoethanol) and boiling at $100{ }^{\circ} \mathrm{C}$ for 6 min. Proteins were separated by SDS-PAGE with $8.5 \%$ polyacrylamide gel and transferred to PVDF membrane (Millipore). Membranes were blocked with 5\% skim milk in Tris-buffered saline $(20 \mathrm{mM}$ Tris $\mathrm{pH} 8.0$, and $137.5 \mathrm{mM} \mathrm{NaCl}$ ) with $0.25 \%$ Tween 20 (TBST) for $1 \mathrm{~h}$ and incubated with primary antibodies at $4{ }^{\circ} \mathrm{C}$ overnight and HRP-conjugated secondary antibodies at room temperature for $2 \mathrm{~h}$. Protein signals were detected by ECL solutions (BioRad).

\section{Immunocytochemistry}

Primary cultured cortical neurons at DIV 10 were fixed with $4 \%$ paraformaldehyde and $4 \%$ sucrose in PBS for 15 min and permeabilized with $0.2 \%$ Triton X-100 in PBS for $5 \mathrm{~min}$ and incubated in the blocking solution (3\% BSA in PBS) for $30 \mathrm{~min}$. Neurons were incubated with rabbit anti-FLAG antibody, mouse anti-GFP antibody, or mouse anti- $\alpha$-tubulin antibody diluted in the blocking solution for $2 \mathrm{~h}$ at room temperature or overnight at $4{ }^{\circ} \mathrm{C}$, washed with PBS for three times, followed by incubation with Alexa Fluor 568-conjugated secondary antibody, Alexa Fluor 488-conjugated secondary antibody, Alexa Fluor 647-conjugated secondary antibody, or Alexa Fluor 405-conjugated secondary antibody for $2 \mathrm{~h}$ at room temperature.

Images were obtained using FV3000 confocal laser scanning microscope (Olympus) with UPLSAPO $40 \times / 0.95$ NA objective or UPLSAPO $100 \times / 1.45$ NA oil objective and deconvolved using advanced constrained iterative $(\mathrm{CI})$ algorithm-based deconvolution program of cellSens (Olympus).

\section{Mitochondrial transport imaging and analysis}

Mitochondrial transport imaging was performed following the previous description with modifications $[53,58]$. Primary cultured cortical neurons at DIV 7-9 were transfected with MTS-mCherry and indicated constructs. At DIV 10-12, live time-lapse imaging was performed using FV3000 confocal laser scanning microscope (Olympus) with UPLSAPO $20 \times / 0.75 \mathrm{NA}$ objective at $37{ }^{\circ} \mathrm{C}$ with supplying $5 \% \mathrm{CO}_{2}$ gas. Neurons were imaged for $2 \mathrm{~min}$ with $3 \mathrm{~s}$ interval. The obtained images were subjected to the analyses for the mitochondrial motility using cellSens (Olympus). Based on the morphological criteria, an axon was identified as a long and thin process, while dendrites were shorter and thicker. In axons, a $150 \mu \mathrm{m}$ segment from at least $100 \mu \mathrm{m}$ away from soma was selected as a proximal axon, and a $150 \mu \mathrm{m}$ segment from the axon tip was selected as a distal axon. For the analysis of dendrites, a $100 \mu \mathrm{m}$ segment of the longest dendrite $100 \mu \mathrm{m}$ away from soma was analyzed. Mitochondrion showing a displacement from the original point at least $5 \mu \mathrm{m}$ for 2 min was regarded as a motile one. The motile and stationary mitochondria were counted manually based on 
the image sequences and kymographs generated by cellSens (Olympus). The motility of mitochondria was presented as the percentage of motile mitochondria.

\section{Calcium imaging and analysis}

Live calcium imaging was performed following previous descriptions with modifications [59, 60]. Primary cultured cortical neurons at DIV 7-9 were transfected with cyto-GCaMP6s or vGlut1-GCaMP5G and indicated constructs followed by time-lapse calcium imaging at DIV 10-12. Primary cultured neurons were loaded with low potassium buffer (10 mM HEPES, $\mathrm{pH} 7.4,126 \mathrm{mM}$

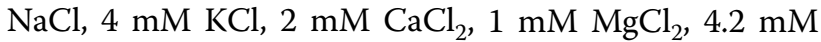
glucose) and treated with $50 \mathrm{mM} \mathrm{KCl}$. The fluorescence intensities were recorded in FV31S-DT software at an interval of $2 \mathrm{~s}$ for total $180 \mathrm{~s}$ for cyto-GCaMP6s and total $120 \mathrm{~s}$ for vGlut1-GCaMP5G using FV3000 confocal laser scanning microscope (Olympus) with UPLSAPO $20 \times / 0.75 \mathrm{NA}$ objective at $37{ }^{\circ} \mathrm{C}$ with supplying $5 \% \mathrm{CO}_{2}$ gas. Background fluorescence was subtracted and the amplitude $\left(\Delta \mathrm{F} / \mathrm{F}_{0}\right)$ of each neuron was calculated as $(\mathrm{F}-$ $\left.\mathrm{F}_{0}\right) / \mathrm{F}_{0}$, where $\mathrm{F}_{0}$ is a baseline GCaMP fluorescence signal averaged over $30 \mathrm{~s}$ before the stimulation and $\mathrm{F}$ is a peak intensity of fluorescence in the response. The trace of calcium decay of each neuron was fit with nonlinear regression using GraphPad Prism. The decay time to halfmaximum was obtained by the half-life of the monoexponential curve.

\section{Microtubule co-sedimentation assay}

Microtubule co-sedimentation assay was carried out as a previous description with modifications [53, 61]. Briefly, brains isolated from WT and Sandy mice were homogenized in microtubule stabilizing buffer $(100 \mathrm{mM}$ PIPES (pH 6.8), 0.1\% NP40, 5 mM MgCl 2,2 mM EGTA, $100 \mathrm{mM} \mathrm{NaF}, 2 \mathrm{M}$ Glycerol, and protease inhibitor cocktail) and centrifuged at $20,000 \times g$ for $40 \mathrm{~min}$ at $4{ }^{\circ} \mathrm{C}$. The supernatant was treated with $30 \mu \mathrm{M}$ Taxol and incubated for $30 \mathrm{~min}$ at $37^{\circ} \mathrm{C}$, followed by centrifugation at $12,000 \times g$ for $40 \mathrm{~min}$ at room temperature. The pellets were resuspended in microtubule stabilizing buffer and supernatant was dissolved in the SDS-PAGE sample buffer and subjected to the western blot analysis.

\section{Mitochondrial fractionation}

Mitochondrial fractionation from primary cultured neurons was performed as a previous description with modifications [53]. Additionally, AraC (\#Cat C1768, Sigma-Aldrich) was added to the neurons at DIV 3 for $24 \mathrm{~h}$ to inhibit the growth of non-neuronal cells. Harvested neurons at DIV 11 were washed with PBS, suspended in mitochondria isolation buffer $(250 \mathrm{mM}$ sucrose, $1 \mathrm{mM}$ EGTA, $1 \mathrm{mM} \mathrm{MgCl}_{2}, 0.5 \mathrm{mM}$ DTT, $10 \mathrm{mM}$ Tris, $\mathrm{pH}$ 8.0), and disrupted by dounce homogenization. The homogenate was spun at $800 \times g$ for $10 \mathrm{~min}$ at $4{ }^{\circ} \mathrm{C}$ twice. The supernatant was recovered and centrifuged again at $8000 \times g$ for $10 \mathrm{~min}$ at $4{ }^{\circ} \mathrm{C}$. The resulting pellet (mitochondrial fraction) was collected, and the supernatant (cytosolic fraction) was cleared by further centrifugation at $12,000 \times g$ for $10 \mathrm{~min}$ at $4{ }^{\circ} \mathrm{C}$.

Mitochondrial fractionation from mouse brain lysates was performed as previously described [62]. A brain was rinsed and homogenized in isolation buffer-1 $(225 \mathrm{mM}$ mannitol, $75 \mathrm{mM}$ sucrose, 0.5\% BSA, $0.5 \mathrm{mM}$ EGTA, $30 \mathrm{mM}$ Tris $-\mathrm{HCl} \mathrm{pH} \mathrm{7.4)} \mathrm{and} \mathrm{centrifuged} \mathrm{at} 740 \times g$ for 5 min at $4{ }^{\circ} \mathrm{C}$. A small portion of the supernatant was kept as a whole lysate fraction and the rest was centrifuged at $9000 \times g$ for $10 \mathrm{~min}$ at $4{ }^{\circ} \mathrm{C}$. The supernatant was kept as a cytosolic fraction, the pellet was resuspended in isolation buffer-2 (225 mM mannitol, $75 \mathrm{mM}$ sucrose, 0.5\% BSA, $30 \mathrm{mM}$ Tris $-\mathrm{HCl} \mathrm{pH} \mathrm{7.4)} \mathrm{and} \mathrm{centrifuge} \mathrm{at} 10,000 \times g$ for $10 \mathrm{~min}$ at $4{ }^{\circ} \mathrm{C}$. The resultant pellet was resuspended in isolation buffer-3 (225 mM mannitol, $75 \mathrm{mM}$ sucrose, $30 \mathrm{mM}$ Tris $-\mathrm{HCl} \mathrm{pH} 7.4)$ and centrifuged at $10,000 \times g$ for $10 \mathrm{~min}$ at $4{ }^{\circ} \mathrm{C}$. The pellet was resuspended in MRB (mitochondria resuspending buffer) $(250 \mathrm{mM}$ mannitol, 5 mM HEPES (pH 7.4), 0.5 mM EGTA) and a small portion was collected as crude mitochondria fraction. This fraction was loaded on percoll medium (225 mM mannitol, 25 mM HEPES (pH 7.4), 1 mM EGTA, 30\% Percoll ( $\mathrm{vol} / \mathrm{vol})$ ) and centrifuged at $95,000 \times g$ for $30 \mathrm{~min}$ at $4{ }^{\circ} \mathrm{C}$ in a Beckman Coulter Optima XE-100 Ultracentrifuge (SW41 Ti rotor). The lower bands were collected and diluted with $\mathrm{MRB}$ and centrifuged at $6,300 \times g$ for $10 \mathrm{~min}$ at $4{ }^{\circ} \mathrm{C}$. The pellet was collected as pure mitochondria fraction and resuspended in MRB.

\section{Statistical analysis}

All graphs were analyzed using the GraphPad Prism software and presented as the mean \pm standard error of mean (SEM). Statistical significance of the data was determined by two-tailed Student's $t$ test for comparisons between two groups and one-way analysis of variance (ANOVA) followed by Bonferroni's post-hoc test for comparisons among multiple groups (Additional files 5, 6).

\section{Supplementary Information}

The online version contains supplementary material available at https://doi. org/10.1186/s13041-020-00720-3.

Additional file 1: Fig. S1. Altered mitochondrial movement and density in axons upon dysbindin knockdown and overexpression. a Quantitative analyses of mitochondrial movement and $\mathbf{b}$ anterograde and retrograde movements in the proximal and distal axons of DIV 10-12 primary cortical 
neurons transfected as indicated. c Quantification of mitochondrial density (the number of mitochondria per $100 \mu \mathrm{m}$ ) in the proximal and distal axons of DIV 10-12 primary cortical neurons ( $n=23$ axons for CTL shRNA, $n=33$ axons for Dysbindin shRNA, and $n=24$ axons for Dysbindin shRNA + mDysbindin for the proximal axons, $n=14$ axons for CTL shRNA, $n=14$ axons for Dysbindin shRNA, and $n=7$ axons for Dysbindin shRNA + mDysbindin for the distal axons). $\mathbf{d}$ Quantitative analyses of mitochondrial movement, and $\mathbf{e}$ anterograde and retrograde movements in the proximal and distal axons of DIV 10-12 primary cortical neurons transfected with FLAG-mDysbindin. $\mathbf{f}$ Quantification of mitochondrial density in the proximal and distal axons of DIV 10-12 primary cortical neurons ( $n=38$ axons for CTL, and $n=37$ axons for mDysbindin for the proximal axons, $n=18$ axons for $C T L$, and $n=21$ axons for mDysbindin for the distal axons). $\mathbf{g}$ Quantitative analysis of mitochondrial movement and $\mathbf{h}$ mitochondrial density in dendrites of DIV 10-12 primary cortical neurons transfected as indicated ( $n=34$ dendrites for CTL shRNA, $n=27$ dendrites for Dysbindin shRNA). All results are presented as the mean \pm SEM. ${ }^{*} p<0.05,{ }^{* *} p<0.01$, and ${ }^{* * *} p<0.001$ from one-way ANOVA with Bonferroni's multiple comparison test for $a, b$, and c, and Student's $t$-test for $d, e, f, g$, and $h$.

Additional file 2: Fig. S2. Detection of endogenous dysbindin and p150 glued from the mitochondrial fraction and reduced interaction of p150 glued-dynamitin upon dysbindin knockdown. a Endogenous dysbindin was detected from the mitochondrial fraction of mouse brain lysates by western blotting with anti-dysbindin antibody. Mitofilin and GAPDH were used as markers for the mitochondrial and cytosolic fractions, respectively. b The protein level of endogenous p $150^{\text {glued }}$ was compared between the mitochondrial fractionation of WT and Sandy mouse brain lysates. Endogenous p150 glued was detected by western blotting with anti-p150 glued antibody. Mitofilin and a-tubulin were used as markers for the mitochondrial and cytosolic fractions, respectively. c Knockdown of endogenous dysbindin by human dysbindin shRNA in HEK293 cells. d Quantification of the protein level of dysbindin normalized by GAPDH. e Co-immunoprecipitation of p $150^{\text {glued }}$ and dynamitin upon dysbindin knockdown. Lysates from HEK293 cells transfected with the indicated shRNA were immunoprecipitated with anti-p150glued antibody. Immunoprecipitates were analyzed by western blotting with anti-dynamitin and anti-p150 glued $\mathbf{f}$ Quantification of the protein level of co-immunoprecipitated dynamitin normalized by immunoprecipitated $\mathrm{p} 15 \mathrm{~g}^{\text {glued }}$. Asterisks indicate the protein of interest. All results are presented as the mean \pm SEM. ${ }^{*} p<0.05,{ }^{* *} p<0.01$, and ${ }^{* * *} p<0.001$ from Student's $t$-test.

Additional file 3: Fig. S3. Mitochondrial density in the proximal axon and mitochondrial motility in the distal axon affected by p150glued and dysbindin. a Quantification of mitochondrial density in the proximal axons of DIV 10-12 primary cortical neurons transfected as indicated $(n=32$ axons for CTL shRNA, $n=33$ axons for p150 glued shRNA, $n=14$ axons for Dysbindin shRNA, and $n=24$ axons for $p 150^{\text {glued }}$ shRNA + Dysbindin shRNA). $\mathbf{b}$ Quantitative analysis of mitochondrial movement in the distal axons of DIV 10-12 primary cortical neurons ( $n=26$ axons for CTL shRNA, $n=12$ axons for $p 150^{\text {glued }}$ shRNA, $n=9$ axons for Dysbindin shRNA, and $\mathrm{n}=17$ axons for $150^{\text {glued }}$ shRNA + Dysbindin shRNA). c Quantification of mitochondrial density in the proximal axons of DIV 10-12 primary cortical neurons transfected as indicated $(n=34$ axons for CTL shRNA, $n=30$ axons for $p 150^{\text {glued }}$ shRNA, $n=32$ axons for mDysbindin, and $n=33$ axons for p150 glued shRNA + mDysbindin). d Quantitative analysis of mitochondrial movement in the distal axons of DIV 10-12 primary cortical neurons ( $n=49$ axons for CTL shRNA, $n=27$ axons for p150 glued shRNA, $n=34$ axons for mDysbindin, and $\mathrm{n}=30$ axons for $150^{\text {glued }}$ shRNA + mDysbindin). All results are presented as the mean \pm SEM. ${ }^{*} p<0.05,{ }^{* *} p<0.01$, and ${ }^{* * *} p<0.001$ from one-way ANOVA with Bonferroni's multiple comparison test.

Additional file 4: Fig. S4. Calcium dynamics in presynaptic terminals of Sandy neurons and presynaptic boutons of dysbindin knockdown neurons. Live-cell calcium imaging for monitoring calcium dynamics. a Calcium response graph obtained after $50 \mathrm{mM} \mathrm{KCl} \mathrm{stimulation} \mathrm{in} \mathrm{DIV} \mathrm{10-12}$ primary cortical neurons expressing cyto-GCaMP6 of WT and Sandy mice. b Statistically analyzed peak amplitudes, $\mathbf{c}$ the area under the curves, and d the decay time to half-maximum of the calcium response graphs $(n=45$ for WT and $\mathrm{n}=51$ for Sandy). e Calcium response graph obtained after $50 \mathrm{mM} \mathrm{KCl}$ stimulation in DIV 10-12 primary cortical neurons expressing vGlut1-GCaMP5 and the indicated shRNA. f Statistically analyzed peak amplitudes and $\mathbf{g}$ the area under the curves ( $n=92$ for CTL shRNA and $\mathrm{n}=134$ for Dysbindin shRNA). All results are presented as the mean \pm SEM. ${ }^{*} p<0.05,{ }^{* *} p<0.01$, and ${ }^{* *} p<0.001$ from Student's $t$-test.

Additional file 5: Raw data - quantified numerical data.

Additional file 6: Raw data - raw images of western blotting.

\section{Abbreviations}

Aß: Amyloid $\beta$; ALS: Amyotrophic lateral sclerosis; ANOVA: Analysis of variance; AP-3: Adaptor related protein complex-3; BLOC-1: Biogenesis of lysosomerelated organelle complex 1; CAP-Gly: Cytoskeleton-associated protein glycine-rich; CTL: Control; DISC1: Disrupted-in-schizophrenia 1; DIV: Days in vitro; IC: Intermediate chain; EGFP: Enhanced green fluorescent protein; EM: Electron microscopy; ER: Endoplasmic reticulum; GABA: Gamma-aminobutyric acid; GFP: Green fluorescent protein; HEK: Human embryonic kidney; IP: Immunoprecipitation; KCl: Potassium chloride; MTS: Mitochondrial transit sequence; SBMA: Spinobulbar muscular atrophy; SEM: Standard error of mean; shRNA: Short hairpin ribonucleic acid; WT:Wild-type.

\section{Acknowledgements}

We thank Dr. Koh-ichi Nagata (Institute for Developmental Research, Aichi Human Service Center, Kasugai, Japan) for providing dysbindin antibody. We thank Dr. Seok-Kyu Kwon (Korea Institute of Science and Technology, Republic of Korea) for providing vGlut1-GCaMP5G.

\section{Authors' contributions}

BKS conceived the study, performed experiments, analyzed and interpreted data, and wrote the manuscript; SAL, CP made initial observations and provided critical ideas for experiments; YS, SJK, YW, TTMN, SBL, DJM, BSG performed experiments and revised the manuscript, HSC, SJK performed experiments; SKP conceived the study, interpreted data, wrote the manuscript, and supervised the project. All authors read and approved the final manuscript.

\section{Funding}

This work was supported by the Advanced Research Center Program (Organelle Network Research Center, 2017R1A5A1015366), the Brain Research Program (2017M3C7A1047875), and the Bio \& Medical Technology Development Program (2020M3E5E2039894) funded by Korean National Research Foundation (S.K.P). This work was also supported by the Global Ph.D. Fellowship (2012H1A2A1016264) funded by Korean National Research Foundation (B.K.S).

\section{Availability of data and materials}

The data generated or analyzed during this study are included in this published article and its supplementary information files.

\section{Ethics approval and consent to participate}

All animal procedures were approved by the Institutional Animal Care and Use Committee (IACUC) of Pohang University of Science and Technology (POSTECH-2019-0024). All experiments were carried out in accordance with the approved guidelines.

\section{Consent for publication}

\author{
Not applicable.
}

\section{Competing interests}

The authors declare no competing interests.

\section{Author details}

${ }^{1}$ Department of Life Sciences, Pohang University of Science and Technology, Pohang, Republic of Korea. ${ }^{2}$ Weill Institute of Neurosciences, Department of Neurology, University of California, San Francisco, San Francisco, USA. ${ }^{3}$ Department of Chemical Engineering, Pohang University of Science and Technology, Pohang, Republic of Korea. 
Received: 14 September 2020 Accepted: 23 December 2020

Published online: 18 January 2021

\section{References}

1. Lin MY, Sheng ZH. Regulation of mitochondrial transport in neurons. Exp Cell Res. 2015;334(1):35-44.

2. Barlan K, Gelfand VI. Microtubule-based transport and the distribution, tethering, and organization of organelles. Cold Spring Harb Perspect Biol. 2017:9(5):a025817.

3. Schroer TA. Dynactin. Annu Rev Cell Dev Biol. 2004;20:759-79.

4. Urnavicius L, Zhang K, Diamant AG, Motz C, Schlager MA, Yu M, et al. The structure of the dynactin complex and its interaction with dynein. Science. 2015;347(6229):1441-6.

5. King SJ, Schroer TA. Dynactin increases the processivity of the cytoplasmic dynein motor. Nat Cell Biol. 2000;2(1):20-4.

6. Ayloo S, Lazarus JE, Dodda A, Tokito M, Ostap EM, Holzbaur EL. Dynactin functions as both a dynamic tether and brake during dynein-driven motility. Nat Commun. 2014;5:4807.

7. Yu J, Lai C, Shim H, Xie C, Sun L, Long CX, et al. Genetic ablation of dynactin p150(Glued) in postnatal neurons causes preferential degeneration of spinal motor neurons in aged mice. Mol Neurodegener. 2018;13(1):10.

8. Roy S, Zhang B, Lee VM, Trojanowski JQ. Axonal transport defects: a common theme in neurodegenerative diseases. Acta Neuropathol. 2005;109(1):5-13.

9. Reddy PH, Tripathi R, Troung Q, Tirumala K, Reddy TP, Anekonda V, et al. Abnormal mitochondrial dynamics and synaptic degeneration as early events in Alzheimer's disease: implications to mitochondria-targeted antioxidant therapeutics. Biochim Biophys Acta. 2012;1822(5):639-49.

10. Pozo Devoto VM, Falzone TL. Mitochondrial dynamics in Parkinson's disease: a role for alpha-synuclein? Dis Model Mech. 2017;10(9):1075-87.

11. Kung $L$, Roberts RC. Mitochondrial pathology in human schizophrenic striatum: a postmortem ultrastructural study. Synapse. 1999;31(1):67-75.

12. Somerville SM, Conley RR, Roberts RC. Mitochondria in the striatum of subjects with schizophrenia. World J Biol Psychiatry. 2011;12(1):48-56.

13. Roberts RC, Barksdale KA, Roche JK, Lahti AC. Decreased synaptic and mitochondrial density in the postmortem anterior cingulate cortex in schizophrenia. Schizophr Res. 2015;168(1-2):543-53.

14. Jimerson DC, Post RM, Carman JS, van Kammen DP, Wood JH, Goodwin FK, et al. CSF calcium: clinical correlates in affective illness and schizophrenia. Biol Psychiatry. 1979;14(1):37-51.

15. Lidow MS. Calcium signaling dysfunction in schizophrenia: a unifying approach. Brain Res Brain Res Rev. 2003;43(1):70-84.

16. Park SJ, Jeong J, Park YU, Park KS, Lee H, Lee N, et al. Disrupted-in-schizophrenia-1 (DISC1) regulates endoplasmic reticulum calcium dynamics. Sci Rep. 2015;5:8694.

17. Park SJ, Lee SB, Suh Y, Kim SJ, Lee N, Hong JH, et al. DISC1 modulates neuronal stress responses by gate-keeping ER-mitochondria $\mathrm{Ca}(2+)$ transfer through the MAM. Cell Rep. 2017;21(10):2748-59.

18. Benson MA, Newey SE, Martin-Rendon E, Hawkes R, Blake DJ. Dysbindin, a novel coiled-coil-containing protein that interacts with the dystrobrevins in muscle and brain. J Biol Chem. 2001;276(26):24232-41.

19. Dickman DK, Davis GW. The schizophrenia susceptibility gene dysbindin controls synaptic homeostasis. Science. 2009;326(5956):1127-30.

20. Jia JM, Hu Z, Nordman J, Li Z. The schizophrenia susceptibility gene dysbindin regulates dendritic spine dynamics. J Neurosci. 2014;34(41):13725-36.

21. Mullin AP, Sadanandappa MK, Ma W, Dickman DK, VijayRaghavan K, Ramaswami M, et al. Gene dosage in the dysbindin schizophrenia susceptibility network differentially affect synaptic function and plasticity. J Neurosci. 2015;35(1):325-38.

22. Wentzel C, Delvendahl I, Sydlik S, Georgiev O, Muller M. Dysbindin links presynaptic proteasome function to homeostatic recruitment of low release probability vesicles. Nat Commun. 2018;9(1):267.

23. Talbot $\mathrm{K}$, Eidem WL, Tinsley $\mathrm{CL}$, Benson MA, Thompson EW, Smith RJ, et al. Dysbindin-1 is reduced in intrinsic, glutamatergic terminals of the hippocampal formation in schizophrenia. J Clin Invest. 2004;113(9):1353-63.

24. Weickert CS, Straub RE, McClintock BW, Matsumoto M, Hashimoto R, Hyde TM, et al. Human dysbindin (DTNBP1) gene expression in normal brain and in schizophrenic prefrontal cortex and midbrain. Arch Gen Psychiatry. 2004;61(6):544-55.

25. Scheggia D, Mastrogiacomo R, Mereu M, Sannino S, Straub RE, Armando $M$, et al. Variations in Dysbindin-1 are associated with cognitive response to antipsychotic drug treatment. Nat Commun. 2018;9(1):2265.

26. Karlsgodt KH, Robleto K, Trantham-Davidson H, Jairl C, Cannon TD, Lavin A, et al. Reduced dysbindin expression mediates $N$-methyl-D-aspartate receptor hypofunction and impaired working memory performance. Biol Psychiatry. 2011;69(1):28-34.

27. Glen WB Jr, Horowitz B, Carlson GC, Cannon TD, Talbot K, Jentsch JD, et al. Dysbindin-1 loss compromises NMDAR-dependent synaptic plasticity and contextual fear conditioning. Hippocampus. 2014;24(2):204-13.

28. Feng YQ, Zhou ZY, He X, Wang H, Guo XL, Hao CJ, et al. Dysbindin deficiency in sandy mice causes reduction of snapin and displays behaviors related to schizophrenia. Schizophr Res. 2008;106(2-3):218-28.

29. Papaleo F, Yang F, Garcia S, Chen J, Lu B, Crawley JN, et al. Dysbindin-1 modulates prefrontal cortical activity and schizophrenia-like behaviors via dopamine/D2 pathways. Mol Psychiatry. 2012;17(1):85-98.

30. Zhang W, Daly KM, Liang B, Zhang L, Li X, Li Y, et al. BDNF rescues prefrontal dysfunction elicited by pyramidal neuron-specific DTNBP1 deletion in vivo. J Mol Cell Biol. 2017;9(2):117-31.

31. Talbot K, Cho DS, Ong WY, Benson MA, Han LY, Kazi HA, et al. Dysbindin-1 is a synaptic and microtubular protein that binds brain snapin. Hum Mol Genet. 2006;15(20):3041-54.

32. Kubota K, Kumamoto N, Matsuzaki S, Hashimoto R, Hattori T, Okuda H, et al. Dysbindin engages in c-Jun N-terminal kinase activity and cytoskeletal organization. Biochem Biophys Res Commun. 2009;379(2):191-5.

33. Mead CL, Kuzyk MA, Moradian A, Wilson GM, Holt RA, Morin GB. Cytosolic protein interactions of the schizophrenia susceptibility gene dysbindin. J Neurochem. 2010;113(6):1491-503.

34. Dickman DK, Tong A, Davis GW. Snapin is critical for presynaptic homeostatic plasticity. J Neurosci. 2012;32(25):8716-24.

35. Di Giovanni J, Sheng ZH. Regulation of synaptic activity by snapin-mediated endolysosomal transport and sorting. EMBO J. 2015;34(15):2059-77.

36. Talbot K, Ong WY, Blake DJ, Tang J, Louneva N, Carlson GC, et al. Dysbindin-1 and its protein family. In: Lajtha A, editor., et al., Handbook of neurochemistry and molecular neurobiology. Boston: Springer; 2009. p. 107-241.

37. Xu Y, Sun Y, Ye H, Zhu L, Liu J, Wu X, et al. Increased dysbindin-1B isoform expression in schizophrenia and its propensity in aggresome formation. Cell Discov. 2015;1:15032.

38. Lee SA, Kim SM, Suh BK, Sun HY, Park YU, Hong JH, et al. Disrupted-inschizophrenia 1 (DISC1) regulates dysbindin function by enhancing its stability. J Biol Chem. 2015;290(11):7087-96.

39. Lee A, Hirabayashi Y, Kwon SK, Lewis TL Jr, Polleux F. Emerging roles of mitochondria in synaptic transmission and neurodegeneration. Curr Opin Physiol. 2018;3:82-93.

40. Guedes-Dias P, Holzbaur ELF. Axonal transport: driving synaptic function. Science. 2019;366:6462.

41. Starcevic M, Dell'Angelica EC. Identification of snapin and three novel proteins (BLOS1, BLOS2, and BLOS3/reduced pigmentation) as subunits of biogenesis of lysosome-related organelles complex-1 (BLOC-1). J Biol Chem. 2004;279(27):28393-401.

42. Larimore J, Tornieri K, Ryder PV, Gokhale A, Zlatic SA, Craige B, et al. The schizophrenia susceptibility factor dysbindin and its associated complex sort cargoes from cell bodies to the synapse. Mol Biol Cell. 2011;22(24):4854-67.

43. $\mathrm{Pu} J$, Schindler $C$, Jia R, Jarnik M, Backlund P, Bonifacino JS. BORC, a multisubunit complex that regulates lysosome positioning. Dev Cell. 2015:33(2):176-88.

44. Kwon SK, Sando R 3rd, Lewis TL, Hirabayashi Y, Maximov A, Polleux F. LKB1 regulates mitochondria-dependent presynaptic calcium clearance and neurotransmitter release properties at excitatory synapses along cortical axons. PLoS Biol. 2016;14(7):e1002516.

45. Vaccaro V, Devine MJ, Higgs NF, Kittler JT. Miro1-dependent mitochondrial positioning drives the rescaling of presynaptic $\mathrm{Ca}^{2+}$ signals during homeostatic plasticity. EMBO Rep. 2017;18(2):231-40.

46. Chen XW, Feng YQ, Hao CJ, Guo XL, He X, Zhou ZY, et al. DTNBP1, a schizophrenia susceptibility gene, affects kinetics of transmitter release. J Cell Biol. 2008;181(5):791-801. 
47. Jentsch JD, Trantham-Davidson H, Jairl C, Tinsley M, Cannon TD, Lavin A. Dysbindin modulates prefrontal cortical glutamatergic circuits and working memory function in mice. Neuropsychopharmacology. 2009;34(12):2601-8.

48. Yuan $Q$, Yang F, Xiao Y, Tan S, Husain N, Ren M, et al. Regulation of brain-derived neurotrophic factor exocytosis and gamma-aminobutyric acidergic interneuron synapse by the schizophrenia susceptibility gene dysbindin-1. Biol Psychiatry. 2016;80(4):312-22.

49. Trantham-Davidson H, Lavin A. Loss of dysbindin-1 affects GABAergic transmission in the PFC. Psychopharmacology. 2019;236(11):3291-300.

50. Carlsson A, Waters N, Holm-Waters S, Tedroff J, Nilsson M, Carlsson ML. Interactions between monoamines, glutamate, and GABA in schizophrenia: new evidence. Annu Rev Pharmacol Toxicol. 2001;41:237-60.

51. Lewis DA, Moghaddam B. Cognitive dysfunction in schizophrenia: convergence of gamma-aminobutyric acid and glutamate alterations. Arch Neurol. 2006;63(10):1372-6.

52. Norkett R, Modi S, Birsa N, Atkin TA, Ivankovic D, Pathania M, et al. DISC1dependent regulation of mitochondrial dynamics controls the morphogenesis of complex neuronal dendrites. J Biol Chem. 2016;291 (2):613-29.

53. Park C, Lee SA, Hong JH, Suh Y, Park SJ, Suh BK, et al. Disrupted-inschizophrenia 1 (DISC1) and syntaphilin collaborate to modulate axonal mitochondrial anchoring. Mol Brain. 2016;9(1):69.

54. Swank RT, Sweet HO, Davisson MT, Reddington M, Novak EK. Sandy: a new mouse model for platelet storage pool deficiency. Genet Res. 1991;58(1):51-62.

55. Ito H, Morishita R, Shinoda T, Iwamoto I, Sudo K, Okamoto K, et al. Dysbindin-1, WAVE2 and Abi-1 form a complex that regulates dendritic spine formation. Mol Psychiatry. 2010;15(10):976-86.
56. Chen TW, Wardill TJ, Sun Y, Pulver SR, Renninger SL, Baohan A, et al. Ultrasensitive fluorescent proteins for imaging neuronal activity. Nature. 2013;499(7458):295-300

57. Lazarus JE, Moughamian AJ, Tokito MK, Holzbaur EL. Dynactin subunit p150(Glued) is a neuron-specific anti-catastrophe factor. PLoS Biol. 2013;11(7):e1001611.

58. Wang X, Schwarz TL. Imaging axonal transport of mitochondria. Methods Enzymol. 2009;457:319-33.

59. Grienberger $C$, Konnerth A. Imaging calcium in neurons. Neuron. 2012;73(5):862-85.

60. Bootman MD, Rietdorf K, Collins T, Walker S, Sanderson M. Ca ${ }^{2+}$-sensitive fluorescent dyes and intracellular $\mathrm{Ca}^{2+}$ imaging. Cold Spring Harb Protoc. 2013;2013(2):83-99.

61. Souphron J, Bodakuntla S, Jijumon AS, Lakisic G, Gautreau AM, Janke $C$, et al. Purification of tubulin with controlled post-translational modifications by polymerization-depolymerization cycles. Nat Protoc. 2019;14(5):1634-60.

62. Wieckowski MR, Giorgi C, Lebiedzinska M, Duszynski J, Pinton P. Isolation of mitochondria-associated membranes and mitochondria from animal tissues and cells. Nat Protoc. 2009;4(11):1582-90.

\section{Publisher's Note}

Springer Nature remains neutral with regard to jurisdictional claims in published maps and institutional affiliations.
Ready to submit your research? Choose BMC and benefit from:

- fast, convenient online submission

- thorough peer review by experienced researchers in your field

- rapid publication on acceptance

- support for research data, including large and complex data types

- gold Open Access which fosters wider collaboration and increased citations

- maximum visibility for your research: over 100M website views per year

At BMC, research is always in progress.

Learn more biomedcentral.com/submissions 